\title{
Vascular heterogeneity between native rat pancreatic islets is responsible for differences in survival and revascularisation post transplantation
}

\author{
Sara Ullsten • Joey Lau $\cdot$ Per-Ola Carlsson
}

Received: 18 June 2014 / Accepted: 1 September 2014 / Published online: 26 September 2014

(C) Springer-Verlag Berlin Heidelberg 2014

\begin{abstract}
Aims/hypothesis Highly blood-perfused islets have been observed to be the most functional islets in the native pancreas. We hypothesised that differences in vascular support of islets in donor pancreases influence their susceptibility to cellular stress and capacity for vascular engraftment after transplantation.

Methods Highly blood-perfused islets in rats were identified by injection of microspheres into the ascending aorta before islet isolation. Cell death was evaluated after in vitro cytokine or hypoxia exposure, and 2 days post transplantation. One month post transplantation, islet engraftment, including vascular density, blood perfusion and oxygen tension $\left(p \mathrm{O}_{2}\right)$ in the tissue, was evaluated.

Results Microsphere-containing islets had a similar frequency of cell death during standard culture conditions but increased cell death after exposure to cytokines and hypoxia in comparison with other islets. Two days after transplantation the percentage of apoptotic or necrotic cells was also higher in grafts of such islets and 1 month post transplantation these grafts were composed of substantially more connective tissue. Grafts of highly blood-perfused islets in the native pancreas regained a higher vascular density, blood perfusion and $p \mathrm{O}_{2}$ in comparison with grafts of other islets.

Conclusions/interpretation Native islets that are highly blood-perfused regained this feature after transplantation, indicating a superior capacity for revascularisation and posttransplant function. However, the same group of islets was
\end{abstract}

S. Ullsten $\cdot$ J. Lau $\cdot$ P.-O. Carlsson $(\bowtie)$

Department of Medical Cell Biology, Uppsala University,

Husargatan 3, Box 571, SE-75123 Uppsala, Sweden

e-mail: per-ola.carlsson@mcb.uu.se

P.-O. Carlsson

Department of Medical Sciences, Uppsala University,

Uppsala, Sweden more vulnerable to different kinds of cellular stress, which limited their early survival post transplantation. Preferential death of these most active islets may contribute to the high number of islets needed to provide cure with islet transplantation.

Keywords Engraftment · Heterogeneity · Islet transplantation · Islet vascularity

\section{Abbreviations \\ PI Propidium iodide \\ $p \mathrm{O}_{2} \quad$ Oxygen tension}

\section{Introduction}

Islet transplantation provides a possibility to restore normoglycaemia in patients with type 1 diabetes. However, the long-term results have been hampered by substantial early cell death due to hypoxic [1] and inflammatory events [2], and later to insufficient engraftment [3, 4], gluco-/lipotoxicity [5], islet amyloid formation [6, 7] and immunological events [8]. The exaggerated cell death occurring in the islet grafts necessitates transplantation of large numbers of islets to restore and maintain normoglycaemia in the recipients, which is largely problematic when considering the worldwide lack of organs for transplantation.

One aspect previously not considered in the outcome of islet transplantation is the heterogeneous nature of the pancreatic islets. Heterogeneities ranging from subpopulations of differently metabolically active beta cells to islet and pancreatic regional heterogeneities have been described [9-14]. Beta cells are also heterogeneous in their capabilities for production of the vasoactive factor serotonin during pregnancy [15]. We have previously observed that differences in islet vascular 
support provide a hallmark that reflects islet functionality. A low-oxygenated proportion of islets were found to be a resting or dormant population, which at increased metabolic demands could be activated and manifested as an increase in oxygen support [10]. Islets that in the native pancreas are highly blood-perfused were on the other hand observed to be the most functionally active, with higher glucose-stimulated insulin release, higher beta cell proliferation and a different gene expression in comparison with size-matched control islets [9].

The present study tested the hypothesis that differences in vascular support, and thereby functional activity, between islets in the native pancreas influence their fate of survival and capacity to engraft after transplantation.

\section{Methods}

Experimental animals Adult male inbred Lewis rats, purchased from Charles River Laboratories (Sulzfeld, Germany), were used. The animals were kept in a specific non-pathogen environment and had free access to water and pelleted food throughout the course of the experiment. 'Principles of laboratory animal care' (National Institutes of Health, Bethesda, MD, USA; publication no. 85-23, revised 1985; http:/grants1.nih.gov/grants/olaw/references/phspol. $\mathrm{htm}$, accessed 1 January 2012) were followed, as well as specific national laws where applicable. All experimental procedures were approved by the Animals Ethics Committee of Uppsala University.

Microsphere administration The rats were anaesthetised by an i.p. injection of $120 \mathrm{mg} / \mathrm{kg}$ body weight thiobutabarbital sodium (Inactin; Sigma-Aldrich, St Louis, MO, USA) and placed on a thermostat-controlled heating plate to maintain a core body temperature of $38^{\circ} \mathrm{C}$. The trachea was detached from surrounding tissues and a polyethylene catheter was inserted to secure free airways during the experiment. Polyethylene catheters were also inserted into the ascending aorta through the right carotid artery for blood pressure measurement and subsequent microsphere injection, and into the femoral artery for reference blood sample collection. After the surgical procedure, the blood pressure was allowed to stabilise for $10-15 \mathrm{~min}$; $1 \mathrm{ml}$ of $30 \%$ (wt/vol.) D-glucose was then injected into the femoral vein $10 \mathrm{~min}$ before fluorescent microsphere injection $(10 \mu \mathrm{m}$, FluoSpheres Polystyrene Microspheres; Molecular Probes, Eugene, OR, USA) [16, 17]. A reference blood sample was collected during $1 \mathrm{~min}$, starting $5 \mathrm{~s}$ before the microsphere injection, in order to determine the blood flow each microsphere represented. The pancreas was removed for islet isolation and the adrenal glands were examined to evaluate adequate mixing of microspheres in the systemic circulation. Only in animals with equal amounts of microspheres in the adrenal glands $(<10 \%$ difference), indicating a successful mix of microspheres with the arterial blood, was the pancreas processed for islet isolation.

Islet isolation Islets were isolated by collagenase digestion, as described previously [18], and thereafter hand-picked. Islets were cultured free-floating and dispersed in $50 \mathrm{~mm}$ Petri dishes (100 islets per dish; Sterilin, Newport, UK) in RPMI 1640 medium supplemented with L-glutamine (SigmaAldrich), benzylpenicillin (100 U/ml; Roche Diagnostics Scandinavia, Bromma, Sweden), streptomycin $(0.1 \mathrm{mg} / \mathrm{ml}$; Sigma-Aldrich) and 10\% (vol./vol.) FCS (Sigma-Aldrich) for 3 days. Using a fluorescence microscope the islets were then sorted dichotomously into two groups, with or without microspheres. Each experiment was if possible performed on islets from one rat. However, pooling of islets from more than one rat was often necessary, and then each rat contributed with the same percentage of microsphere- and non-microspherecontaining islets.

Cellular stress in vitro In each experiment 100 microspherecontaining or non-microsphere-containing islets were assigned to each of two groups. This resulted in a total of 200 analysed islets per experiment. The microspherecontaining islets were in each experiment matched by size (median diameter) and number with non-microspherecontaining islets from the same rat. Visible islet aggregations were excluded. The islets were incubated in $20 \%$ or $1.5 \% \mathrm{O}_{2}$ in a humidified airtight chamber for $4 \mathrm{~h}$ at $37^{\circ} \mathrm{C}$. In separate experiments, the islets were incubated with or without cytokines $(50 \mathrm{U} / \mathrm{ml} \mathrm{IL}-1 \beta, 1,000 \mathrm{U} / \mathrm{ml} \mathrm{IFN}-\gamma, 1,000 \mathrm{U} / \mathrm{ml}$ TNF- $\alpha$; PeproTech, London, UK) for $24 \mathrm{~h}$. In the former experiments, gas mixtures were produced by a certified manufacturer (Air Liquid Gas, Stockholm, Sweden), and the oxygen levels were continuously monitored with an oxygen sensor (Dräger Pac III; Dräger, Lübeck, Germany) in order to maintain correct oxygen tension $\left(p \mathrm{O}_{2}\right)$ exposure during the experiments. Viability of the islets was evaluated by staining with propidium iodide (PI) $(10 \mu \mathrm{g} / \mathrm{ml}$; Sigma-Aldrich) and bisbenzimide $(20 \mu \mathrm{g} / \mathrm{ml}$, Hoechst 33342; Sigma-Aldrich). Fluorescence was analysed in a Kodak Image Station 4000 MM (Kodak, New Haven, CT, USA). The ratio of PI to bisbenzimide was taken as a relative measure of islet viability.

Islet transplantation The rats were anaesthetised by an i.p. injection of $60 \mathrm{mg} / \mathrm{kg}$ body weight of pentobarbital sodium (Apoteket, Umeå, Sweden) and placed on a thermostatcontrolled heating plate to maintain a core body temperature of $38^{\circ} \mathrm{C}$. One hundred microsphere- and 100 nonmicrosphere-containing islets were transplanted separately at a distance $10-15 \mathrm{~mm}$ apart beneath the left renal capsule. Two days or 1 month post transplantation the graft-bearing kidneys were removed. Two hours before removal of the 2-day-old 
grafts the hypoxia marker pimonidazole hydrochloride (60 mg/kg body weight, Hypoxyprobe-1; HPI, Burlington, MA, USA) was intravenously injected. The grafts were fixed in $10 \%$ (vol./vol.) buffered formalin, dehydrated and embedded in paraffin. Embedded tissues were consecutively sectioned (5 $\mu \mathrm{m}$ thick) and mounted on Polysine slides (Thermo Scientific, Braunschweig, Germany).

Immunohistochemistry Sections from the islet grafts were deparaffinised with xylene and rehydrated in a series of graded ethanol $(100 \%$ to $70 \%)$ followed by distilled water, or deparaffinised by Rodent Decloaker (Biocare Medical, Concord, CA, USA) in a pressure cooker (2100 Retriever; Prestige Medical, Blackburn, UK). Islet grafts retrieved 2 days post transplantation were stained for apoptosis and hypoxia markers, whereas islet grafts retrieved 1 month post transplantation were stained for insulin and endothelial cells. Pretreatment for hypoxia staining was performed with Pronase (0.01\%; Roche Diagnostics, Mannheim, Germany), for apoptosis staining modified citrate buffer ( $\mathrm{pH}$ 6.1; Target Retrieval Solution, Dako, Glostrup, Denmark), and for endothelial staining a heat-induction and neuraminidase solution (0.1 U/ml, Neuroamidase Type V; Sigma-Aldrich) was used [19], followed by peroxidase blocking in 3\% hydrogen peroxidase (hydrogen peroxide 30\%; Merck, Darmstadt, Germany) and protein blocking (TNB solution, TSA Biotin System Kit [Perkin Elmer, Waltham, MA, USA] or Background Sniper [Biocare Medical]). Apoptosis was detected by a cleaved caspase-3 antibody (1:100; Cell Signaling Technology, Danvers, MA, USA). The signal was amplified by a TSA Biotin System Kit (Perkin Elmer) according to the manufacturer's protocol and detected by 3,3'-diaminobenzidine (SigmaAldrich). Rat pancreases were used as controls in order to exclude non-specific antibody binding. Hypoxyprobe-1 MAb1 (1:25; HPI) was used for hypoxia/pimonidazole hydrochloride staining, and biotinylated lectin from Bandeirea simplicifolia agglutinin-1 (1:100; Sigma-Aldrich) for staining of endothelial cells, followed by incubation with TrekAvidinAP Label (Biocare Medical) and detection by Vulcan Fast Red (Biocare Medical). Insulin antibody (1:3,000, polyclonal antiguinea pig insulin; Fitzgerald, Acton, MA, USA) was detected by MACH 3 Rabbit HRP-Polymer Detection (Biocare Medical) and visualised by 3,3'-diaminobenzidine. All sections were counterstained with haematoxylin before mounting.

Analysis Scanning and analysis were performed using a laser microdissection microscope (Leica LMD6000, Leica Microsystems, Wetzlar, Germany) or after digital image acquisition by Image J (National Institutes of Health). Necrotic graft area with structural disintegration of cells (pyknotic nuclei, cellular debris) was assessed by morphological evaluation, where on average a total graft area of $0.65 \pm 0.1 \mathrm{~mm}^{2}$ per animal was evaluated in the analysis. Apoptosis rate was defined as the percentage of cells positively stained for cleaved caspase-3, where on average $2,841 \pm 258$ islet cells per group of islets and animal were evaluated. Hypoxic area was defined as the area positively stained for the hypoxia marker pimonidazole per total endocrine tissue area, where an endocrine area of $0.63 \pm 0.1 \mathrm{~mm}^{2}$ per animal was evaluated. Vascular density was defined as the area of stained endothelial cells per insulin-positive area. The total insulin-positive area analysed per animal was $0.54 \pm$ $0.06 \mathrm{~mm}^{2}$. Connective tissue area was evaluated by morphometry and defined as non-endocrine tissue per total graft area, where $1.42 \pm 0.1 \mathrm{~mm}^{2}$ total graft area per animal was evaluated.

Measurements of islet graft blood flow and $\mathrm{pO}_{2}$ The animals were anaesthetised and surgically prepared according to a protocol similar to that used for microsphere administration. Additional procedures included the insertion of a femoral vein catheter for substitution of body fluid loss with Ringer's solution (Apoteket), and exposure of the graft-bearing kidney by a left subcostal flank incision. The kidney was thereafter placed in a plastic cup and embedded in surgical cotton pads soaked in saline $(154 \mathrm{mmol} / \mathrm{l} \mathrm{NaCl})$ in order to stabilise and maintain humidity and temperature of the organ. The blood flow and $p \mathrm{O}_{2}$ in the islet grafts and adjacent renal parenchyma were then recorded by laser Doppler flowmetry and Clark microelectrodes, respectively [20]. Multiple (three or more) measurements of blood flow by laser Doppler flowmetry (Transonic BLF21 Series, probe $1.2 \mathrm{~mm}$; Transonic, Ithaca, NY, USA) were performed in each of the two grafts and in the kidney cortex. Similarly, the $p \mathrm{O}_{2}$ in each of the islet grafts and adjacent kidney was investigated by repeated measurements (three or more in each of the locations) by Clark microelectrodes (outer tip diameter $<5 \mu \mathrm{m}$; Unisense, Aarhus, Denmark). The mean of blood flow or $p \mathrm{O}_{2}$ values in each location and animal was then calculated and considered to be one experiment. Since it is difficult to calibrate the laser Doppler flowmetry instrument in physical units of blood flow, all blood flow values are given as arbitrary tissue perfusion units.

Statistical analysis All values are given as means \pm SEM. Differences between two groups of parametric data were analysed by unpaired or paired two-tailed Student's $t$ test, whereas for multiple comparisons of parametric data a twoway ANOVA with Bonferroni's or Dunnett's (comparisons only to control) post hoc test was applied; $p$ values $<0.05$ were considered statistically significant.

\section{Results}

Microsphere administration The animals allocated to intraaortic microsphere administration all had a mean arterial blood 
pressure of $100-125 \mathrm{mmHg}(n=57)$. These animals received $1 \mathrm{ml} \mathrm{30 \%}$ (wt/vol.) D-glucose and had a plasma glucose concentration of $10.4 \pm 0.3 \mathrm{mmol} / 1$ at the time of microsphere administration $10 \mathrm{~min}$ later. Islet isolation resulted in an average of 500 isolated islets per rat, where $13.9 \pm 0.8 \%$ of the islets contained one or more microspheres (Fig. 1). Each microsphere was calculated to correspond on average to $0.52 \pm 0.04 \mu \mathrm{l} / \mathrm{min}$ blood.

Cellular stress in vitro No difference in cell death could be seen between islets with or without microspheres during standard culture conditions (control; Fig. 2). However, exposure to hypoxic conditions $\left(1.5 \% \mathrm{O}_{2}\right)$ augmented the cell death by approximately $40 \%$ in islets with microspheres in comparison with non-microsphere-containing islets under similar conditions (Fig. 2a). Similarly, after exposure to cytokines the cellular death was $\sim 50 \%$ higher in islets with microspheres (Fig. 2a). The cellular death in the hypoxia- and cytokineexposed microsphere-containing islets was increased to the same extent in the immediate vicinity (within a radius of $50 \mu \mathrm{m})$ of the microspheres $(109 \pm 19 \%$ and $100 \pm 11 \%$, respectively, of that in the whole islets; $n=6$ experiments), as in other parts of the islets.

Cellular stress in vivo The grafts were severely hypoxic 2 days after transplantation, where on average $61 \pm 3 \%$ of the grafts of islets with or without microspheres were positively stained for the hypoxia marker pimonidazole (Fig. 3a). Necrotic areas, with pyknotic nuclei or cellular debris, were regularly observed surrounded by viable hypoxic tissue (Fig. 3a). The relative area of the endocrine grafts composed of such necrotic areas was doubled in the grafts of islets with microspheres in comparison with grafts of islets without microspheres 2 days

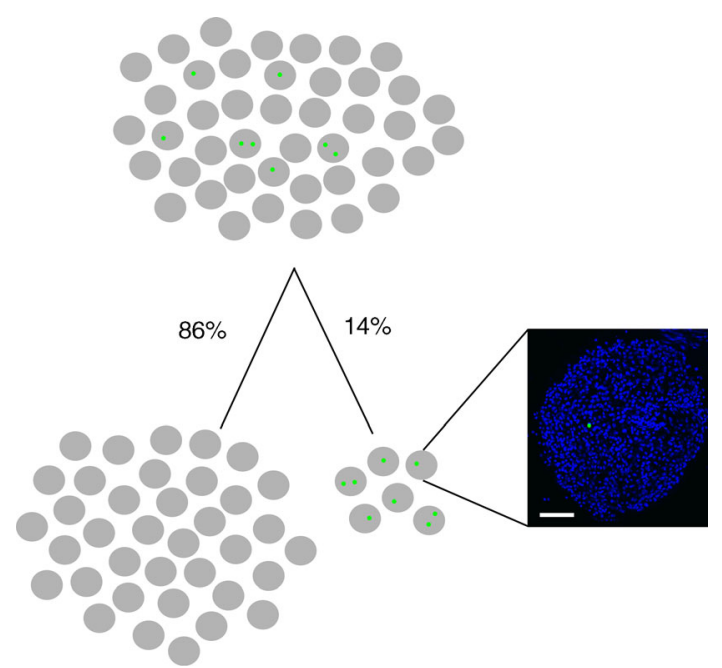

Fig. 1 Schematic outline of the sorting of microsphere- and nonmicrosphere-containing islets. Scale bar, $100 \mu \mathrm{m}$
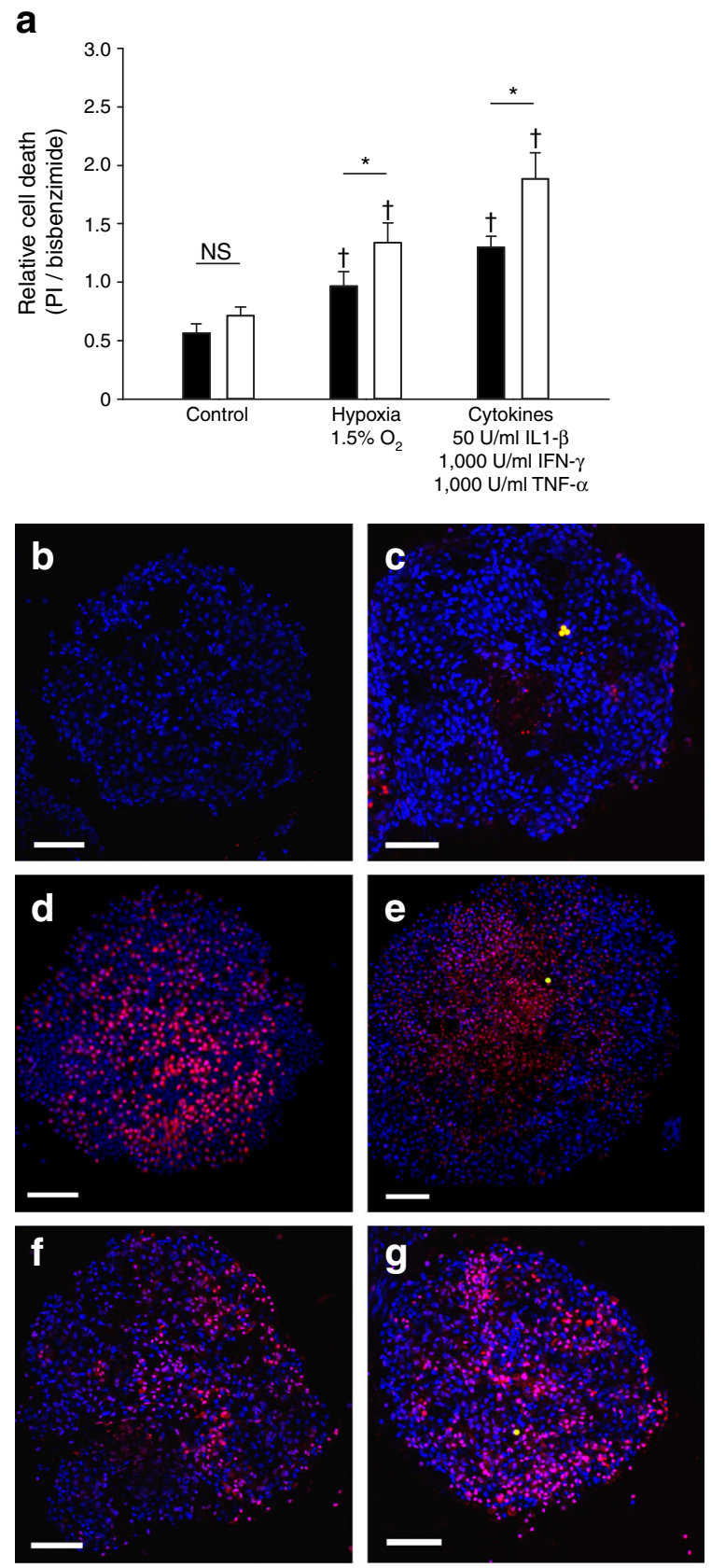

Fig. 2 Cellular death in vitro in microsphere- and nonmicrosphere-containing islets. (a) Relative cell death as measured by $\mathrm{PI} /$ bisbenzimide ratios in non-microsphere-containing islets (black bars) and in microsphere-containing islets (white bars) during standard culture conditions (control), or after exposure to hypoxia $\left(1.5 \% \mathrm{O}_{2}\right)$ or cytokines $(50 \mathrm{U} / \mathrm{ml} \mathrm{IL}-1 \beta, 1,000 \mathrm{U} / \mathrm{ml}$ IFN- $\gamma, 1,000 \mathrm{U} / \mathrm{ml}$ TNF- $\alpha$ ). All values are given as means \pm SEM for between eight and ten experiments (each experiment based on islets from at least one animal). ${ }^{*} p<0.05$ vs nonmicrosphere-containing islets; ${ }^{\dagger} p<0.05$ vs corresponding islets during standard culture conditions. Panels $\mathbf{b}-\mathbf{g}$ show cell death in non-microsphere-containing islets and microspherecontaining islets under standard culture conditions (b and c, respectively), during hypoxic conditions (d and e, respectively), and after exposure to cytokines ( $\mathbf{f}$ and $\mathbf{g}$, respectively). PI is shown in red, bisbenzimide in blue, and microspheres (c, e and $\mathbf{g}$ ) in yellow. Scale bar, $100 \mu \mathrm{m}$ 

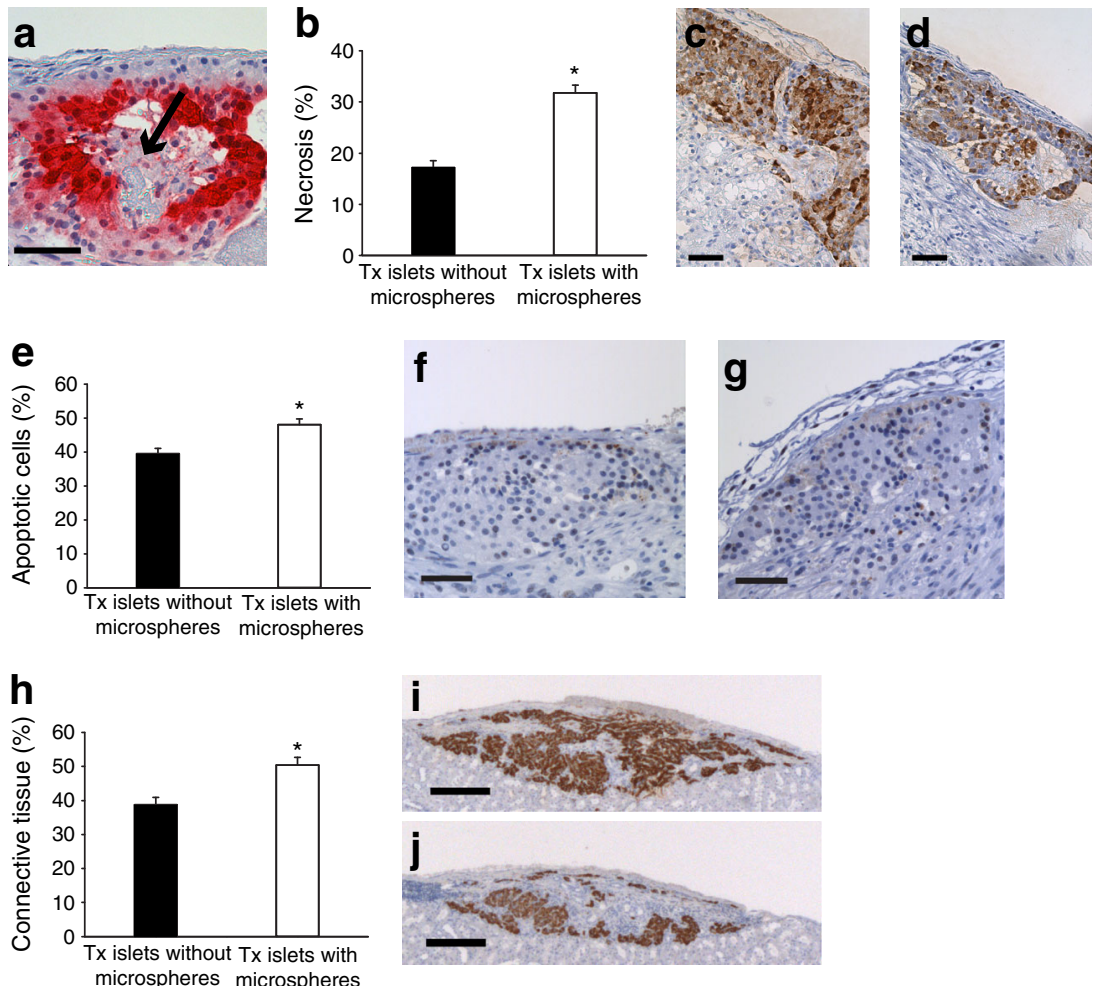

Fig. 3 Hypoxia and cellular death after islet transplantation. (a) Hypoxia was evaluated by pimonidazole staining (red) in islet grafts 2 days post transplantation. Necrosis (arrow) was commonly seen in the central parts of the grafts surrounded by pimonidazole-positive areas; scale bar, $50 \mu \mathrm{m}$. (b-d) Two days after islet transplantation necrosis was more common in islet grafts of microsphere-containing islets (d) than in grafts of non-microsphere-containing islets (c); insulin is shown in brown; scale bar, $50 \mu \mathrm{m}$. (e- g) Similarly, 2 days after islet transplantation apoptosis was more common in islet grafts of microsphere-containing islets $(\mathbf{g})$ than

after transplantation (Fig. 3b-d). Moreover, the number of endocrine cells undergoing apoptosis was also higher in grafts of islets with microspheres (Fig. 3e-g). One month post transplantation, the grafts of islets with microspheres contained $\sim 30 \%$ more connective tissue than the grafts of islets without microspheres (Fig. 3h-j).

Vascular engraftment The mean vascular density in the insulin-positive area of the islets was more than 50\% higher in grafts of islets with microspheres than in grafts of nonmicrosphere-containing islets (Fig. $4 \mathrm{a}-\mathrm{c}$ ). The higher vascular density seemed to reflect functional blood vessels, since this was paralleled by a $\sim 50 \%$ higher blood flow in grafts of microsphere-containing islets compared with grafts of islets without microspheres (Fig. 5a). Moreover, the $\mathrm{pO}_{2}$ in the transplanted microsphere-containing islets was substantially higher than that in the corresponding non-microspherecontaining islets (Fig. 5b). All animals in which blood flow and $p \mathrm{O}_{2}$ measurements were investigated had a mean arterial blood pressure between 80 and $125 \mathrm{mmHg}$. in grafts of non-microsphere-containing islets (f); cleaved caspase-3 is shown in brown; scale bar, $50 \mu \mathrm{m}$. (h-j) One month post transplantation, morphometrical analysis showed that there was more connective tissue in islet grafts of microsphere-containing islets (j) than in grafts of nonmicrosphere-containing islets (i); insulin is shown in brown; scale bar, $200 \mu \mathrm{m}$. All values are given as means \pm SEM for six islet grafts of microsphere- or non-microsphere-containing islets, respectively. ${ }^{*} p<0.05$ vs transplanted (Tx) islets without microspheres

\section{Discussion}

The present study shows that the previously described pool of highly functional, highly blood-perfused islets in the native pancreas [9] has a higher susceptibility to cellular stress with
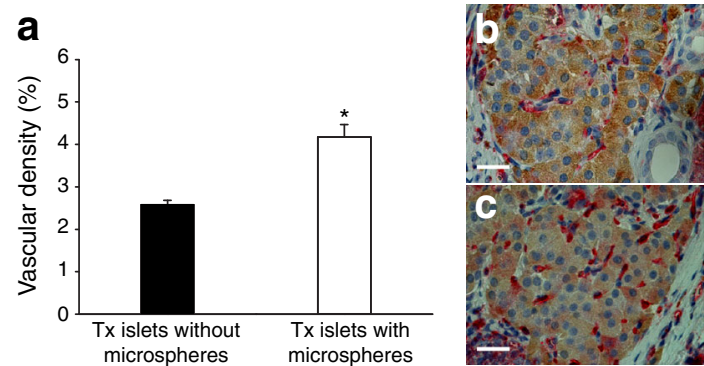

Fig. 4 Vascular density in islet grafts 1 month post transplantation. (a-c) Microsphere-containing islets (c) regained a higher vascular density than non-microsphere-containing islets (b) post transplantation; insulin is shown in brown, endothelial cells in red; scale bar, $50 \mu \mathrm{m}$. Values are given as means \pm SEM for six islet grafts of microsphere- or nonmicrosphere-containing islets, respectively. ${ }^{*} p<0.05$ vs transplanted (Tx) islets without microspheres 
a

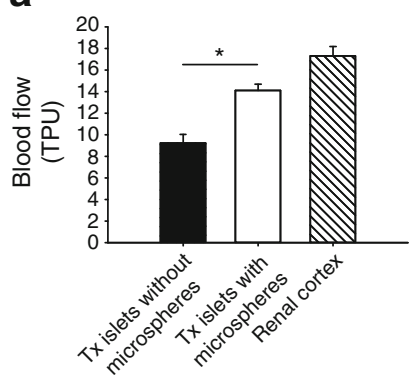

b

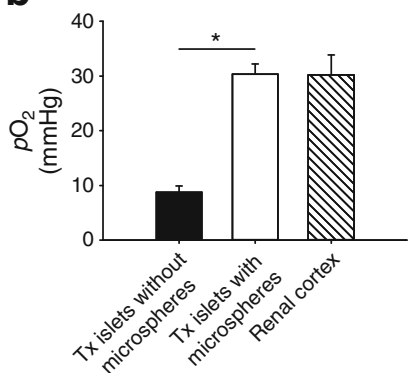

Fig. 5 Blood flow and $p \mathrm{O}_{2}$ in islet grafts 1 month post transplantation. Microsphere-containing islets regained both a higher blood flow (a) and $p \mathrm{O}_{2}$ (b) after transplantation beneath the renal capsule. Measurements were also performed in the renal cortex as a reference organ. All values are given as means \pm SEM for six islet grafts of microsphere- or nonmicrosphere-containing islets. Renal cortex measurements were performed in all six animals. ${ }^{*} p<0.05$ vs transplanted (Tx) islets without microspheres. TPU, tissue perfusion units

resulting death. This bears potential implications for the development of islet disease and holds relevance for islet transplantation outcome. Indeed, the same islets that show propensity for cellular death, if they survive, become better engrafted.

The subpopulation of highly blood-perfused islets in the native pancreas was, as previously described [9], identified by its microsphere content after intra-aortic microsphere administration following a glucose challenge. In the present study, $\sim 14 \%$ of the isolated islets were found to contain microspheres, which, based on the reference blood sample analysis, indicated an average blood perfusion of $\geq 0.52 \pm 0.04 \mu \mathrm{l} / \mathrm{min}$. The microsphere content per se does not seem to elicit harmful effects on the islets, since these islets are characterised by better glucose-stimulated insulin release indices and higher beta cell proliferation than other islets [9]. In line with this, we did not in the present study observe any difference in cellular death between the microsphere-containing and nonmicrosphere-containing islets during standard culture conditions. However, when challenged by cytokines in vitro, the microsphere-containing islets showed a higher propensity for cellular death. The tested cytokines, IL- $1 \beta$, IFN- $\gamma$ and TNF- $\alpha$, have all been shown to play an important role in beta cell death in the development of type 1 diabetes and early after islet transplantation [21-26]. The microsphere-containing islets also showed a higher susceptibility to cellular death in response to hypoxia, which is a major limiting factor of islet survival early post transplantation $[1,27]$. Although it cannot be fully excluded that the microsphere presence made the tissue more sensitive to hypoxic or cytokine-induced death, it is worthy of note that the cellular death was increased to the same extent in the other parts of the islets as in the immediate vicinity of the microspheres.

Early after transplantation up to $60 \%$ of the islet tissue will undergo necrosis and apoptosis due to hypoxia and inflammatory reactions [28-31]. By transplantation of islet grafts of

similar size, but composed of either microsphere- or nonmicrosphere-containing islets, we tested whether our mechanistic in vitro studies were applicable to the in vivo setting. Indeed, higher cellular death by apoptosis and necrosis was observed in the grafts of microsphere-containing islets, which was followed by extensive fibrosis of these grafts. These results are consistent with studies in other research fields, where it has been shown that tissues and cells with a high metabolic rate are more susceptible to cellular stress and death $[32,33]$.

It is of note that the surviving microsphere-containing islets were much better revascularised than the non-microspherecontaining islets. This is likely explained by an inherent difference in gene expression. We have previously observed that these two types of islets markedly differ in their gene expression, which includes higher expression of vascular endothelial growth factor $\mathrm{A}$ in the microsphere-containing islets [9]. Indeed, this growth factor has in a number of studies been shown to be the key mechanism for the development and maintenance of a high islet vascular density [34-36], and it has also been observed to be of central importance for the revascularisation process post transplantation [35, 37]. We have previously investigated only implanting microspheres, and thereby excluded the possibility of increased vascularisation triggered by the microspheres that merely reflect a foreign body reaction [38]. In fact, the most prominent visible difference between the two groups of islets in the present study was the amount of blood vessels penetrating from the surrounding connective tissue into the islet tissue. In the non-microspherecontaining islet grafts, the islet tissue was mostly surrounded by vessels and only a small amount of vessels penetrating the endocrine tissue were observed; this is in comparison with the microsphere-containing islets, which had a large amount of vessels penetrating the endocrine tissue.

The functional capacity of the newly formed blood vessels was investigated by blood flow and $p \mathrm{O}_{2}$ measurements, which both corresponded well to the higher vascular density seen in the grafts of microsphere-containing islets. We recorded an approximately $50 \%$ higher blood flow in the grafts of microsphere-containing islets than in the grafts of nonmicrosphere-containing islets, and an even more substantial difference in islet $p \mathrm{O}_{2}$. The latter indicated a vascular distribution in the microsphere-containing islet grafts that was highly efficient for oxygen delivery to the islet cells and that almost restored tissue oxygenation to the levels previously recorded in native islets $[20,30]$. At present, it remains to be determined whether highly blood perfused islets, despite containing more dying cells, might still regulate blood glucose levels in a better or similar way to those that are less well perfused.

In conclusion, the subpopulation of most active islets in the native pancreas was in the present study observed to be more susceptible to cellular death when exposed to cytokines or 
hypoxia. These islets also had a higher cellular death early after transplantation with resulting extensive fibrosis formation in the grafts. The findings emphasise the importance of developing efficient interventions against cell death early after transplantation, since the most functionally active islets then preferentially die. Surviving islets in this population are shown to become much better engrafted than other islets and will thereby substantially contribute to graft function post transplantation.

Acknowledgements We gratefully acknowledge the skilled technical assistance of A. Nordin and M. Quach (Department of Medical Cell Biology, Uppsala University, Uppsala, Sweden). Some of the data in this work were presented as an abstract at the International Pancreas and Islet Transplantation Association meeting in 2013.

Funding The study was generously supported by the Swedish Research Council (5XX-15043), the Swedish Diabetes Association, the Novo Nordisk Foundation, AFA Insurances, the Olle Engkvist Byggmästare Fund, the Magnus Bergvalls Foundation, the Swedish Juvenile Diabetes Foundation, the Fredrik and Ingrid Thuring Foundation and Diabetes Wellness Sverige.

Duality of interest The authors declare that there is no duality of interest associated with this manuscript.

Contribution statement SU, JL and P-OC conceived and designed the study, and contributed to the acquisition, analysis and interpretation of the data. SU and P-OC drafted the manuscript and JL revised it critically for intellectual content. All authors approved the final version of the paper. $\mathrm{SU}$ is the guarantor of this work.

\section{References}

1. Olsson R, Olerud J, Pettersson U, Carlsson PO (2011) Increased numbers of low-oxygenated pancreatic islets after intraportal islet transplantation. Diabetes 60:2350-2353

2. Bennet W, Sundberg B, Groth CG et al (1999) Incompatibility between human blood and isolated islets of Langerhans: a finding with implications for clinical intraportal islet transplantation? Diabetes 48:1907-1914

3. Lau J, Carlsson PO (2009) Low revascularization of human islets when experimentally transplanted into the liver. Transplantation 87 : 322-325

4. Henriksnas J, Lau J, Zang G, Berggren PO, Kohler M, Carlsson PO (2012) Markedly decreased blood perfusion of pancreatic islets transplanted intraportally into the liver: disruption of islet integrity necessary for islet revascularization. Diabetes 61:665-673

5. Lee Y, Ravazzola M, Park BH, Bashmakov YK, Orci L, Unger RH (2007) Metabolic mechanisms of failure of intraportally transplanted pancreatic beta-cells in rats: role of lipotoxicity and prevention by leptin. Diabetes 56:2295-2301

6. Westermark P, Eizirik DL, Pipeleers DG, Hellerstrom C, Andersson A (1995) Rapid deposition of amyloid in human islets transplanted into nude mice. Diabetologia 38:543-549

7. Westermark GT, Westermark P, Berne C, Korsgren O, Nordic Network for Clinical Islet T (2008) Widespread amyloid deposition in transplanted human pancreatic islets. N Engl J Med 359:977-979

8. Abreu JR, Roep BO (2013) Immune monitoring of islet and pancreas transplant recipients. Curr Diabetes Rep 13:704-712
9. Lau J, Svensson J, Grapensparr L, Johansson A, Carlsson PO (2012) Superior beta cell proliferation, function and gene expression in a subpopulation of rat islets identified by high blood perfusion. Diabetologia 55:1390-1399

10. Olsson R, Carlsson PO (2011) A low-oxygenated subpopulation of pancreatic islets constitutes a functional reserve of endocrine cells. Diabetes 60:2068-2075

11. Schuit FC, In't Veld PA, Pipeleers DG (1988) Glucose stimulates proinsulin biosynthesis by a dose-dependent recruitment of pancreatic beta cells. Proc Natl Acad Sci U S A 85:3865-3869

12. Ellenbroek JH, Tons HA, de Graaf N et al (2013) Topologically heterogeneous beta cell adaptation in response to high-fat diet in mice. PLoS One 8:e56922

13. Katsuta H, Aguayo-Mazzucato C, Katsuta R et al (2012) Subpopulations of GFP-marked mouse pancreatic beta-cells differ in size, granularity, and insulin secretion. Endocrinology 153:5180 5187

14. Van Schravendijk CF, Kiekens R, Pipeleers DG (1992) Pancreatic beta cell heterogeneity in glucose-induced insulin secretion. J Biol Chem 267:21344-21348

15. Schraenen A, Lemaire K, de Faudeur G et al (2010) Placental lactogens induce serotonin biosynthesis in a subset of mouse beta cells during pregnancy. Diabetologia 53:2589-2599

16. Jansson L, Hellerstrom C (1983) Stimulation by glucose of the blood flow to the pancreatic islets of the rat. Diabetologia 25:45-50

17. Iwase M, Tashiro K, Uchizono Y, Goto D, Yoshinari M (2001) Pancreatic islet blood flow in conscious rats during hyperglycemia and hypoglycemia. Am J Physiol Regul Integr Comp Physiol 280: R1601-R1605

18. Andersson A (1978) Isolated mouse pancreatic islets in culture: effects of serum and different culture media on the insulin production of the islets. Diabetologia 14:397-404

19. Mattsson G, Carlsson PO, Olausson K, Jansson L (2002) Histological markers for endothelial cells in endogenous and transplanted rodent pancreatic islets. Pancreatology 2:155-162

20. Carlsson PO, Liss P, Andersson A, Jansson L (1998) Measurements of oxygen tension in native and transplanted rat pancreatic islets. Diabetes 47:1027-1032

21. Mandrup-Poulsen T (1996) The role of interleukin-1 in the pathogenesis of IDDM. Diabetologia 39:1005-1029

22. Cetkovic-Cvrlje M, Eizirik DL (1994) TNF-alpha and IFN-gamma potentiate the deleterious effects of IL-1 beta on mouse pancreatic islets mainly via generation of nitric oxide. Cytokine 6:399-406

23. Kolb H, Luckemeyer K, Heise T et al (2013) The systemic immune network in recent onset type 1 diabetes: central role of interleukin-1 receptor antagonist (DIATOR Trial). PLoS One 8:e72440

24. Ferreira RC, Guo H, Coulson RM et al (2014) A type I interferon transcriptional signature precedes autoimmunity in children genetically at-risk of type 1 diabetes. Diabetes 67:2538-2550

25. Montolio M, Tellez N, Soler J, Montanya E (2007) Role of blood glucose in cytokine gene expression in early syngeneic islet transplantation. Cell Transplant 16:517-525

26. Mastrandrea L, Yu J, Behrens T et al (2009) Etanercept treatment in children with new-onset type 1 diabetes: pilot randomized, placebocontrolled, double-blind study. Diabetes Care 32:1244-1249

27. Hughes SJ, Davies SE, Powis SH, Press M (2003) Hyperoxia improves the survival of intraportally transplanted syngeneic pancreatic islets. Transplantation 75:1954-1959

28. Biarnes M, Montolio M, Nacher V, Raurell M, Soler J, Montanya E (2002) Beta-cell death and mass in syngeneically transplanted islets exposed to short- and long-term hyperglycemia. Diabetes 51:66-72

29. Emamaullee JA, Shapiro AM (2007) Factors influencing the loss of beta-cell mass in islet transplantation. Cell Transplant 16:1-8

30. Carlsson PO, Palm F, Andersson A, Liss P (2001) Markedly decreased oxygen tension in transplanted rat pancreatic islets irrespective of the implantation site. Diabetes 50:489-495 
31. Eich T, Eriksson O, Sundin A et al (2007) Positron emission tomography: a real-time tool to quantify early islet engraftment in a preclinical large animal model. Transplantation 84: 893-898

32. Cooke MS, Evans MD, Dizdaroglu M, Lunec J (2003) Oxidative DNA damage: mechanisms, mutation, and disease. FASEB J 17:1195-1214

33. Boutilier RG (2001) Mechanisms of cell survival in hypoxia and hypothermia. J Exp Biol 204:3171-3181

34. Kamba T, Tam BY, Hashizume H et al (2006) VEGF-dependent plasticity of fenestrated capillaries in the normal adult microvasculature. Am J Physiol Heart Circ Physiol 290:H560-H576
35. Brissova M, Shostak A, Shiota M et al (2006) Pancreatic islet production of vascular endothelial growth factor-a is essential for islet vascularization, revascularization, and function. Diabetes 55: 2974-2985

36. Lammert E, Gu G, McLaughlin M et al (2003) Role of VEGF-A in vascularization of pancreatic islets. Curr Biol 13:1070-1074

37. Zhang N, Richter A, Suriawinata J et al (2004) Elevated vascular endothelial growth factor production in islets improves islet graft vascularization. Diabetes 53:963-970

38. Mattsson G, Jansson L, Carlsson PO (2002) Decreased vascular density in mouse pancreatic islets after transplantation. Diabetes 51 : $1362-1366$ 